\title{
The Relationship between Neutrophil Lymphocyte Ratio and Platelet Lymphocyte Ratio to Degree of Activity in Systemic Lupus Erythematosus
}

\author{
Irsyadil Fikri' ${ }^{1}$, Zuhrial Zubir ${ }^{2}$, Ananda Wibawanta Ginting ${ }^{2}$ \\ ${ }^{1}$ Department of Internal Medicine, Faculty of Medicine, Universitas Sumatera Utara \\ ${ }^{2}$ Division of Pulmonology and Allergy-Immunology, Department of Internal Medicine, Faculty of Medicine, \\ Universitas Sumatera Utara \\ Corresponding Author: Irsyadil Fikri
}

\begin{abstract}
Background: Systemic lupus erythematosus is a chronic autoimmune inflammatory disease with a wide spectrum of clinical and serological manifestations caused by autoantibody production, complement activation, and immune complex deposition. Several studies have shown that the neutrophil-lymphocyte ratio and the platelet-lymphocyte ratio are closely correlated with systemic lupus erythematosus and its disease activity so that they can be used as diagnostic indicators and monitoring of systemic lupus erythematosus.

Objective: To determine the relationship between the ratio of neutrophil lymphocytes and the ratio of platelets to lymphocytes on the degree of activity of lupus disease in patients with systemic lupus erythematosus.

Methods: This is an observational analytic study using medical record data from central installation patients at H. Adam Malik Hospital in the period January to December 2019. The sample was calculated using the unpaired comparative sample size formula for more than two groups of one measurement. Then the distribution test was carried out with the Shapiro Wilk test. Bivariate analysis was conducted to determine the relationship between the ratio of neutrophil lymphocytes and the ratio of platelets to lymphocytes with the MEX SLEDAI score using the ANOVA test if the data were normally distributed, or the Kruskal-Wallis test if the data was not normally distributed. Then proceed with the Mann-Whitney post hoc test to see which groups have differences. The sum of deviations $(\alpha)$ is 0.05 , statistically significant if $p<0.05$.
\end{abstract}

Results: 120 subjects participated in the study and 33 people $(27.5 \%)$ had mild systemic lupus erythematosus, $47(39.2 \%)$ moderate degrees, and 40 people $(33.3 \%)$ severe degrees.

Conclusion: The neutrophil-lymphocyte ratio and the platelet-lymphocyte ratio are associated with the degree of lupus activity in patients with systemic lupus erythematosus.

Keywords: neutrophil-lymphocyte ratio, platelet lymphocyte ratio, systemic lupus erythematosus

\section{INTRODUCTION}

Systemic lupus erythematosus (SLE) is a chronic autoimmune inflammatory disease with a broad spectrum of clinical and serological manifestations caused by autoantibody production, complement activation, and immune complex deposition ${ }^{[1,2]}$. SLE is spread throughout the world, found in both sexes, races/ethnicities, and age groups. However, the disease is more common in adults, women, and nonCaucasians ${ }^{[3]}$. The incidence of SLE in China is $31-70$ per 100,000 individuals. The Lupus Foundation of America estimates that about 1.5 million cases occur in America and at least 5 million cases occur in the world. Every year it is estimated that there are about 16,000 new cases. SLE is more common in women than men in all age groups, and particularly in the age group range from 15 to 64 years, where the manto-woman ratio is $6: 10$ in reproductive age [4-6]. 
The etiology of SLE is still not known with certainty. Abnormalities of $\mathrm{T}$ cells, B cells, dendritic cells, Fc $\gamma$ receptors, proinflammatory cytokines, complement pathways, and apoptosis play a role in the pathogenesis of SLE ${ }^{[7]}$. Although the pathogenesis of systemic lupus erythematosus remains unclear, the incidence and progression are closely related to immune system dysfunction, especially in the presence of anti-DNA antibodies ${ }^{[8]}$.

Several studies have shown that the neutrophil-lymphocyte ratio is closely correlated with systemic lupus erythematosus and its disease activity so that the neutrophil-lymphocyte ratio can be used as a diagnostic indicator and monitoring of systemic lupus erythematosus ${ }^{[9]}$. Celikbilek et al. (2013) observed that NLR and PLR in peripheral blood are simple Systemic Inflammatory Response (SIR) markers evaluated from blood parameters and showed that NLR has diagnostic value in certain pathologies characterized by systemic or local inflammatory responses such as diabetes mellitus, coronary artery disease, ulcerative colitis and inflammatory arthritis ${ }^{[10]}$. Turkmen et al. (2013) demonstrated that platelets can interact with various cell types, including endothelial cells, T lymphocytes, neutrophils, and mononuclear phagocytes, which causes chronic inflammation, may contribute to the process of atherosclerosis [11].

Research on this topic has been done previously by Hao et al. (2017), who reported an association between NLR and PLR on the degree of activity of systemic lupus erythematosus ${ }^{[12]}$. However, the number of studies included was relatively small. Therefore, this study aimed to correlate NLR and PLR in patients with systemic lupus erythematosus and to correlate NLR and PLR to the systemic lupus erythematosus disease activity index (Mexican SLE disease activity index, MEXSLEDAI).

\section{METHODS}

The design of this study was analytic observational with a retrospective approach. This research was conducted using medical record data from the Inpatient Installation of patients at H. Adam Malik Hospital Medan after obtaining approval from the USU Medical Research Ethics Commission.

The total sample of the study was 120 patients with systemic lupus erythematosus who were treated from January 2019 to December 2019. The selected sample must meet the inclusion criteria, namely (1) aged 18 years, and (2) patients diagnosed with systemic lupus erythematosus according to the 2019 EULAR/ACR criteria and/or SLICC 2012 criteria, while the exclusion criteria were massive stress (severe trauma, surgery, cardiac shock, burns), (2) patients with other autoimmune diseases, (3) patients with haematological malignancies, (4) severe liver cirrhosis (Child pugh C), (5) acute renal failure, (6) history of repeated transfusion, and (7) incomplete medical record data.

Each patient diagnosed with systemic lupus erythematosus was assessed according to the 2019 EULAR/ACR criteria and/or SLICC 2012. Then, these patients were subjected to blood tests and an activity assessment of the degree of systemic lupus erythematosus using the MEX-SLEDAI criteria. Complete blood count was performed using the Rayto RT-7606 Hematology Analyzer.

Univariate analysis was performed to determine the characteristics of SLE patients. Then the distribution test was carried out with the Shapiro Wilk test. Bivariate analysis was conducted to determine the relationship between the ratio of neutrophil lymphocytes and the ratio of platelets to lymphocytes with the MEX SLEDAI score using the ANOVA test if the data were normally distributed, or the Kruskal-Wallis test if the data was not normally distributed. Then proceed with the Mann-Whitney post hoc test to see which groups have differences. In addition, a test 
Irsyadil Fikri et.al. The relationship between neutrophil lymphocyte ratio and platelet lymphocyte ratio to degree of activity in systemic lupus erythematosus.

was carried out to see the correlation between the ratio of neutrophil to lymphocytes and the ratio of platelets to lymphocytes and the correlation of NLR and PLR values with MEX-SLEDAI scoring with Pearson's correlation if the data were normally distributed or Spearman's correlation if the data were not normally distributed. The magnitude of deviation from desired value $(\alpha)$ is 0.05 , statistically significant if $\mathrm{p}<0.05$.

\section{RESULTS}

\section{Characteristics of Research Subjects}

Based on medical records of patients with systemic lupus erythematosus who were hospitalized in inpatient installation at H. Adam Malik Hospital Medan from January 2019 to December 2019. 9632 patients were hospitalized by the Internal Medicine section with 120 study subjects with a diagnosis of systemic lupus erythematosus who met the inclusion and exclusion criteria. The demographic and clinical characteristics of research subjects are presented in Table 1.

Table 1. Characteristics of research subjects

\begin{tabular}{|c|c|}
\hline Characteristics & $\mathrm{N}=120$ \\
\hline \multicolumn{2}{|l|}{ Age, (years) } \\
\hline Average \pm SB & $30.78 \pm 10.67$ \\
\hline Median (minimum-maximum) & $29.0(18.0-76.0)$ \\
\hline \multicolumn{2}{|l|}{ Gender, $\mathrm{n}(\%)$} \\
\hline Men & $8(6.7)$ \\
\hline Women & $112(93.3)$ \\
\hline \multicolumn{2}{|l|}{ Serositis, $\mathrm{n}(\%)$} \\
\hline Yes & $33(27.5)$ \\
\hline No & $87(72.5)$ \\
\hline \multicolumn{2}{|l|}{ Renal impairment, n(\%) } \\
\hline Yes & $34(28.3)$ \\
\hline No & $86(71.7)$ \\
\hline \multicolumn{2}{|l|}{ Arthritis, $\mathrm{n}(\%)$} \\
\hline Yes & $108(90.0)$ \\
\hline No & $12(10,0)$ \\
\hline \multicolumn{2}{|l|}{ Hematologic disorders, $\mathrm{n}(\%)$} \\
\hline Yes & $82(68.3)$ \\
\hline No & $38(31.7)$ \\
\hline \multicolumn{2}{|l|}{ Neurological disorders, $\mathrm{n}(\%)$} \\
\hline Yes & $8(6.7)$ \\
\hline No & $112(93.3)$ \\
\hline \multicolumn{2}{|l|}{$\begin{array}{l}\text { Degree of systemic lupus erythematosus } \\
\text { (MEX-SLEDAI), } \mathrm{n}(\%)\end{array}$} \\
\hline Mild & $33(27.5)$ \\
\hline Moderate & $47(39.2)$ \\
\hline Severe & $40(33.3)$ \\
\hline \multicolumn{2}{|l|}{ ANA (U) } \\
\hline Average \pm SB & $107.80 \pm 81.11$ \\
\hline Median (minimum-maximum) & $\begin{array}{l}115.00 \quad(2.36- \\
324)\end{array}$ \\
\hline \multicolumn{2}{|l|}{ Anti-dsDNA (IU/ml) } \\
\hline Average \pm SB & $537.09 \pm 436.3$ \\
\hline Median (minimum-maximum) & $\begin{array}{l}454.00 \quad(6.10- \\
1970)\end{array}$ \\
\hline
\end{tabular}

Table 2. Characteristics of laboratory examination parameters

\begin{tabular}{|l|l|l|l|l|l|}
\hline Parameter & Mean & Standard Deviation & Median & Minimum value & Maximum value \\
\hline Neutrophils & 5208,72 & 2454.11 & 5078.63 & 829.04 & 12402.69 \\
\hline Lymphocytes & 1378,82 & 788,21 & 1279.85 & 100,17 & 3765,12 \\
\hline Platelets & 260,341 & 111.409 & 251,000 & 47,00 & 651.00 \\
\hline NLR & 5.36 & 5.85 & 3.94 & 1.11 & 47,10 \\
\hline PLR & 247.16 & 187.88 & 198.68 & 52.97 & 1597.28 \\
\hline MEX-SLEDAI scores & 8.59 & 4.56 & 7.00 & 2.00 & 23.00 \\
\hline
\end{tabular}

The Relationship between NLR and PLR with the Degree of Lupus Disease Activity in Systemic Lupus Erythematosus Patients

Table 3. Analysis of the relationship between NLR and PLR on the degree of lupus activity in patients with systemic lupus erythematosus

\begin{tabular}{|l|l|l|l|l|}
\hline \multirow{2}{*}{ Parameter } & \multicolumn{2}{|l|}{ The degree of activity of systemic lupus erythematosus } & \multirow{2}{*}{ p-value } \\
\cline { 2 - 4 } & Mild $(\mathbf{n}=\mathbf{3 3})$ & Moderate $(\mathbf{n}=\mathbf{4 7})$ & Severe $(\mathbf{n}=\mathbf{4 0})$ & \\
\hline NLR & $2.95(1.49-6.30)$ & $3.84(1.11-47.10)$ & $5.09(1.67-34.07)$ & $0.001^{*}$ \\
\hline PLR & $159.50(52.97-407.19)$ & $178.42(68.63-860.99)$ & $275.96(67.78-1529.28)$ & $<0.001 *$ \\
\hline
\end{tabular}

The median ratio of NLR and PLR was increasing concerning the degree of lupus disease activity in patients with systemic lupus erythematosus. Based on the analysis, it was found that there was a statistically significant relationship between the ratio of NLR and PLR between the degrees of systemic lupus erythematosus with $\mathrm{p}<0.05 \quad(\mathrm{p}=0.001)$ and $\mathrm{p}<0.001$, respectively.

Table 4 shows a significant relationship between the NLR ratio and the degree of activity of mild vs severe and moderate vs severe systemic lupus erythematosus with $\mathrm{p}<0.001$ and $\mathrm{p}=0.031$, respectively. There was a significant relationship between PLR ratio on the 
Irsyadil Fikri et.al. The relationship between neutrophil lymphocyte ratio and platelet lymphocyte ratio to degree of activity in systemic lupus erythematosus.

degree of activity of mild vs severe and moderate vs severe systemic lupus erythematosus disease with $\mathrm{p}<0.001$ and $\mathrm{p}=0.001$, respectively.
Table 4. Post-hoc analysis of the relationship of NLR and PLR to each degree of lupus activity in patients with systemic lupus erythematosus

\begin{tabular}{|l|l|l|}
\hline \multirow{4}{*}{ NLR } & SLE degrees & p-value \\
\cline { 2 - 3 } & Mild vs Moderate & 0.127 \\
\cline { 2 - 3 } & Mild vs Severe & $<0.001^{*}$ \\
\cline { 2 - 3 } & Moderate vs Severe & $0.031^{*}$ \\
\hline \multirow{4}{*}{ PLR } & Mild vs Moderate & 0.307 \\
\cline { 2 - 3 } & Mild vs Severe & $<0.001^{*}$ \\
\cline { 2 - 3 } & Moderate vs Severe & $0.001^{*}$ \\
\cline { 2 - 3 } & Mose & \\
\hline
\end{tabular}

$\mathrm{r}=0.620$ * Post-hoc Mann-Whitney test, significant $p<0.05$.

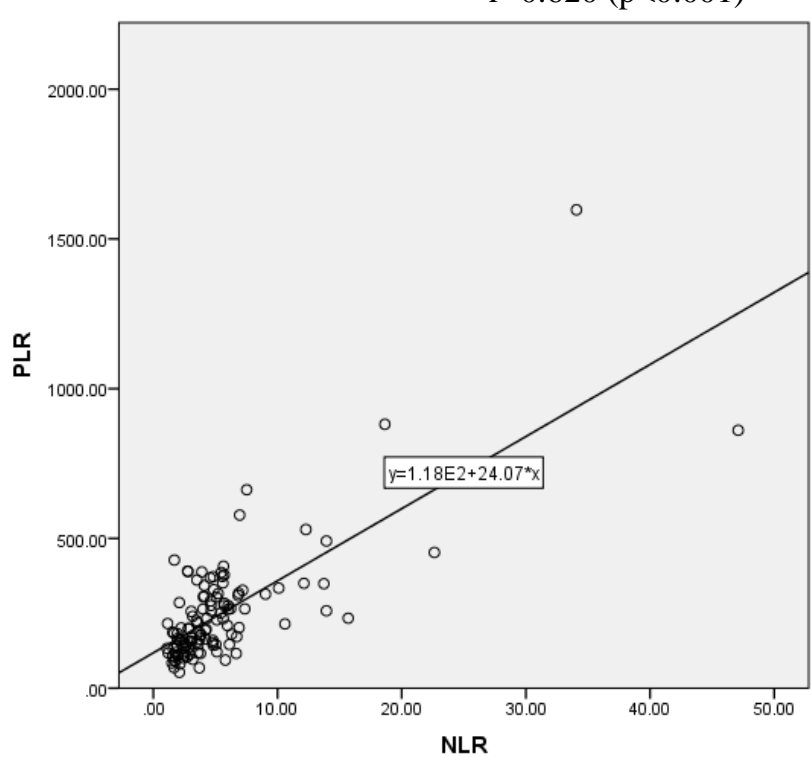

Figure 1. Correlation of NLR and PLR values

Based on Figure 1, it was found that the neutrophil-lymphocyte ratio had a strong positive correlation with the platelet-to-lymphocyte ratio with a value of $r=0.620$, and $p$-value $<0.001$.

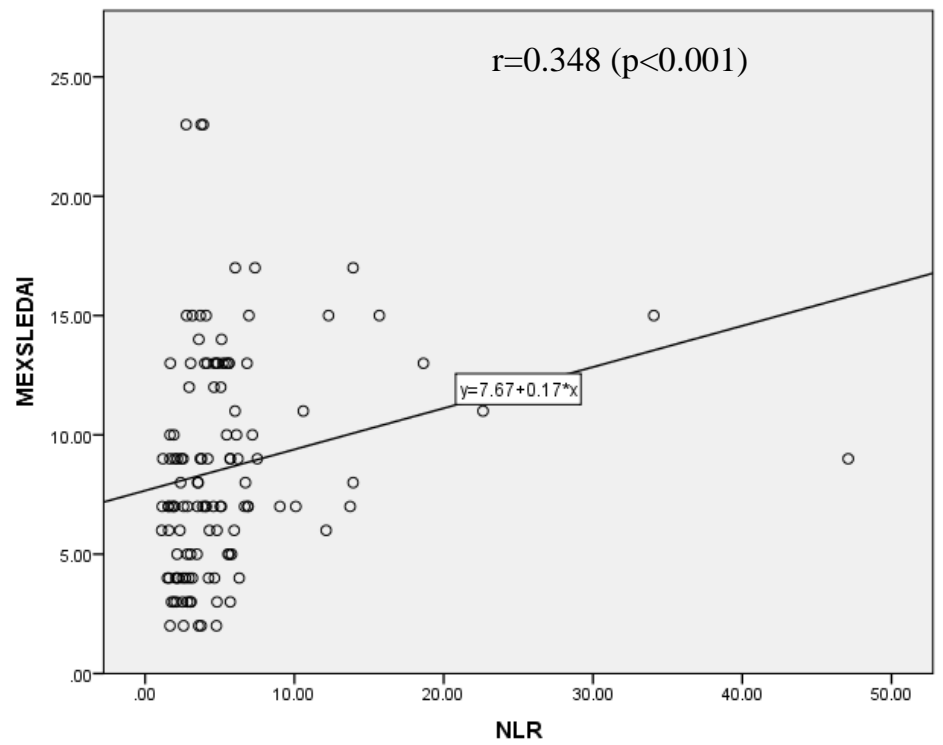

Figure 2. Correlation of NLR scores with MEXSLEDAI scoring

Based on Figure 2, it was found that the neutrophil-lymphocyte ratio had a moderately positive correlation with the MEX-SLEDAI scoring with a value of $r=0.348$, and $p$-value $<0.001$. 


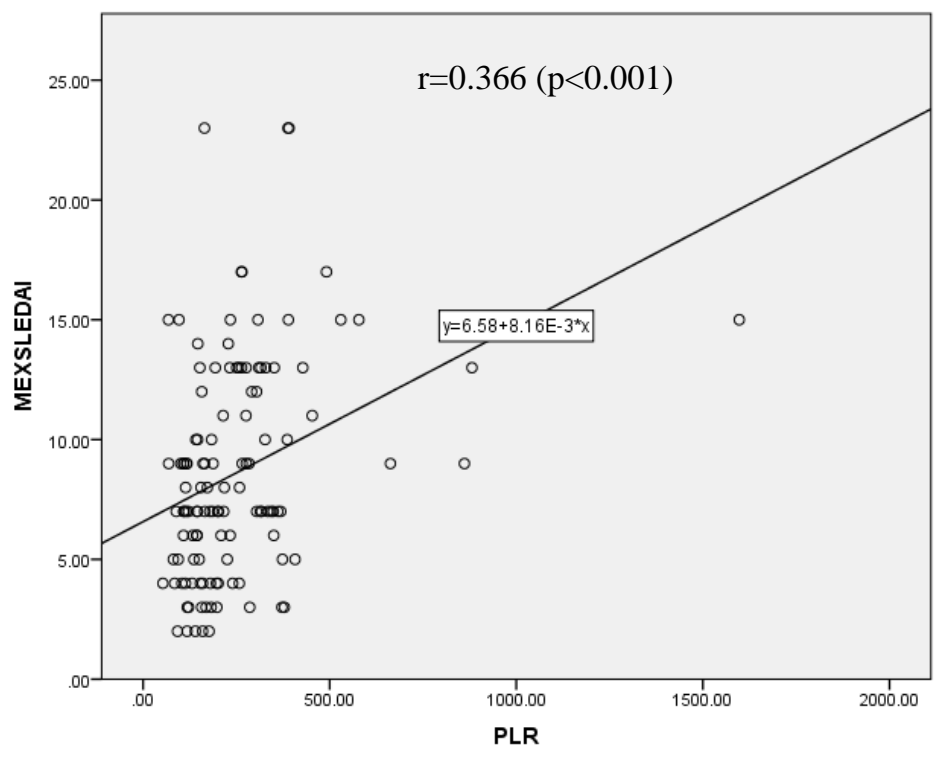

Figure 3. Correlation of PLR scores with MEXSLEDAI scoring

Based on Figure 3, it was found that the platelet-to-lymphocyte ratio had a moderately positive correlation with the MEX-SLEDAI scoring with a value of $\mathrm{r}=0.366$, and $\mathrm{p}$-value $<0.001$.

\section{DISCUSSION}

In this study, the average research subject was 30 years old, with an age range between 18 to 76 years and the majority of research subjects were women, namely 112 people $(93.3 \%)$ and men as many as 8 people $(6.7 \%)$. This is following the study by Rees et al. (2016) who conducted a study on the incidence and prevalence of systemic lupus erythematosus in the UK in the period 1999-2012 and found that systemic lupus erythematosus was six times more common in women $(\mathrm{p}<0.001)$. The age of peak incidence of systemic lupus erythematosus in women (40-49 years) is earlier than in men (60-69 years) ${ }^{[13]}$. The meta-analysis study by Izmirly et al. (2021) on the prevalence of systemic lupus erythematosus in the United States using data from the Centers for Disease Control and Prevention (CDC) National Lupus Registry network of population-based SLE registries found that the prevalence of systemic lupus erythematosus was 9 times higher among women than among women. men (128.7 versus 14.6 per 100,000$)^{[14]}$.
Based on clinical examination, this study found that 33 people with lupus had serositis $(27.5 \%), 34$ people $(28.3 \%)$ had kidney problems, 108 people had arthritis $(90 \%), 82$ hematological disorders. people $(68.3 \%)$, neurological disorders as many as 8 people $(6.7 \%)$. The same result was also obtained by Zian et al. (2018) in 50 lupus erythematosus patients in Morocco, namely arthritis (82\%), mucocutaneous manifestations $(80 \%)$, renal manifestations (50\%), and hematologic features (46\%). From the mucocutaneous characteristics, the highest observed frequencies were malar rash $(68 \%)$ and photosensitivity $(60 \%)$. The central nervous system is involved in $10 \%$ [15].

In this study, the average ANA value was $108 \mathrm{U}$, and the average anti-dsDNA value was $537 \mathrm{IU} / \mathrm{mL}$. The prevalence of positive ANA tests is found in various autoimmune rheumatic diseases, for example, $\quad 90-100 \%$ systemic lupus erythematosus, $60-80 \%$ in systemic sclerosis, $40-70 \%$ in Sjogren's syndrome, $30-80 \%$ in polymyositis/dermatomyositis, and $30-50 \%$ in rheumatoid arthritis (RA) ${ }^{[16]}$. The study by Qu et al. (2018) found that the sensitivity and specificity of ANA in diagnosing SLE were 91.75 and $79.65 \%$; anti-dsDNA antibodies were 67.01 and $98.23 \%$, respectively. Thus, the diagnosis of 
systemic lupus erythematosus with ANA, anti-dsDNA antibodies can be used for the diagnosis and treatment of systemic lupus erythematosus patients ${ }^{[17]}$.

Based on the supporting examination, the mean of neutrophils was $5208.72 \pm 2454.11$ cells $/ \mathrm{mm} 3$, the mean of lymphocytes was $1378.82 \pm 788.21$ cells $/ \mathrm{mm} 3$, the mean of platelets was $260.341 \pm 111,409$ cells $/ \mathrm{mm} 3$. The study by Zian et al. (2018) found that lymphopenia was seen in $30 \%$ of patients, followed by hemolytic anemia in $16 \%$ and leukopenia and thrombocytopenia in $8 \%$ [15]. In the study by Ismunandar et al. (2020) 35 patients with systemic lupus erythematosus found that the mean neutrophil was $5124 \pm 1671.75$, the mean platelet was $262.428 \pm 92629$, and the mean lymphocyte was $1320.18 \pm 782.21^{[18]}$. Hematologic abnormalities are common in systemic lupus erythematosus. Anemia, leukopenia, and thrombocytopenia may occur due to immune-mediated bone marrow depression or excessive peripheral cell destruction. Leukopenia in systemic lupus erythematosus may result from lymphopenia, neutropenia, or both. Neutropenia is a common feature of systemic lupus erythematosus which may be mediated by anti-neutrophil antibodies. Neutrophil dysfunction plays a role in the pathogenesis of systemic lupus erythematosus. The protective function of neutrophils is carried out through various mechanisms: isolation of granular antimicrobial peptides (gAMP), phagocytosis of microbes with degradation via reactive oxygen species in phagolysosomes; and a bactericidal mechanism due to tissue release of chromatin and gAMP. The development of neutropenia in SLE has many causes, including the formation of direct antibodies against leukocytes; which neutralize autoantibodies to neutrophil and cell growth factors - myeloid precursors; bone marrow suppression; involvement of neutrophils in the process of apoptosis and NETosis. Neutrophils in SLE are characterized by decreased phagocytic ability and pathological oxidative activity. In systemic lupus erythematosus, there is a decreased ability to secrete neutrophil apoptotic products, which correlates with disease activity. Other possible causes of hematologic abnormalities in systemic lupus erythematosus are drugs and infection ${ }^{[19,20]}$. A study by Han et al (2020) on 141 systemic lupus erythematosus patients and 79 control patients found that patients with systemic lupus erythematosus had an increase in the number of neutrophils $(p=0.04)$ and a decrease in the number of lymphocytes $\quad(p<0.0001)$, thus causing increase in NLR compared to control $(\mathrm{p}<0.0001)$. Patients with high NLR have active systemic lupus erythematosus [21]. 04) and decreased lymphocyte count $(\mathrm{p}<0.0001)$, causing an increase in NLR compared to control $(\mathrm{p}<0.0001)$. Patients with high NLR have active systemic lupus erythematosus ${ }^{[21]}$. 04) and a decrease in the number of lymphocytes $(\mathrm{p}<0.0001)$, causing an increase in NLR compared to the control $(\mathrm{p}<0.0001)$. Patients with high NLR have active systemic lupus erythematosus ${ }^{[21]}$.

In this study, the majority of study subjects with systemic lupus erythematosus were moderate, namely, 47 people (39.2\%), severe degrees were 40 people (33.3), and mild degrees were 33 people $(27.5 \%)$. In this study, it was found that the average NLR ratio is 5.36, the average PLR ratio is 247.16, and the average MEX-SLEDAI score is 8.59 .

There was a significant relationship between the NLR ratio on the degree of activity of mild vs. severe systemic lupus erythematosus with $p<0.001$ and the degree of activity of moderate-to-severe systemic lupus erythematosus with $\mathrm{p}=0.031$. In the study conducted by Oehadian et al. (2013) in 21 patients with mild and moderate systemic lupus erythematosus according to the MEXSLEDAI criteria and 30 control patients, it was found that there was no significant difference in the neutrophillymphocyte ratio between systemic lupus erythematosus with mild and moderate 
activity $(2.59(1.01-10) .92)$ vs. $2.01(1.38-$ 3.98), $\mathrm{p}=0.412$. [4]. This study also found that there was a significant relationship between the PLR ratio and the degree of activity of mild vs severe systemic lupus erythematosus and moderate vs severe with $\mathrm{p}<0.001$ and $\mathrm{p}=0.001$, respectively.

NLR is a subclinical inflammatory marker that is used in combination with other inflammatory markers to define inflammation in autoimmune and nonautoimmune diseases. PLR is also an index of inflammation in routine blood tests. PLR changes may be related to inflammation and cytokines. The absolute lymphocyte count is usually decreased in autoimmune diseases, and the platelet count is often decreased in patients with systemic lupus erythematosus [22].

The median ratio of NLR and PLR increased with the degree of activity of lupus disease in systemic lupus erythematosus. Based on the analysis, it was obtained that there was a statistically significant relationship between the NLR ratios between groups with the degree of systemic lupus erythematosus with $\mathrm{p}<0.05$ $(\mathrm{p}=0.001)$ and the NLR ratio between groups. systemic lupus erythematosus grade group with $\mathrm{p}<0.001$. In addition, in this study, it was found that the neutrophillymphocyte ratio has a strong positive correlation with the platelet-to-lymphocyte ratio with a value of $r=0.620$, with a value of $p<0.001$. The ratio of neutrophils to lymphocytes and the ratio of platelets to lymphocytes had a moderately positive correlation with the MEX-SLEDAI scoring ( $\mathrm{r}$ NLR=0.348, rPLR=0.366, $\mathrm{p}<0.001$ ).

Several studies have been conducted on the use of NLR and PLR in evaluating the disease activity of systemic lupus erythematosus. In the study by Ismunandar et al. (2020) the mean NLR was 5.37 \pm 4.02 and the mean PLR was $244.26 \pm 113.44$, the mean MEX SLEDAI $=8.63 \pm 5.04$ and NLR and PLR were related to the MEX SLEDAI score $(\mathrm{r} \quad \mathrm{NLR}=0.882, \mathrm{r} \quad \mathrm{PLR}=0.984$, $\mathrm{p}<0.001)^{[18]}$. The study by Qin et al. (2016) in 154 systemic lupus erythematosus patients and 151 control patients, it was found that NLR correlated with the SLE Disease Activity Index (SLEDAI) score (3.61 $\pm 2.04, r=0.471, p<0.01)$. PLR was positively correlated with the SLEDAI score $(155.64 \pm 91.69, r=0.44, p<0.01)^{[22]}$. The study by Soliman et al. (2020) found that NLR and PLR correlated with systemic lupus erythematosus disease activity (median NLR 2.21, $\mathrm{p}=0.024$, median $\mathrm{PLR}=147.5, \mathrm{p}=0.005){ }^{[20]}$. The study by $\mathrm{Li}$ et al. (2015) in 79 patients with systemic lupus erythematosus and 149 healthy patients, it was found that the NLR was significantly higher than the control group (control $=2.00 \pm 0.76$, systemic lupus erythematosus $=4.26 \pm 3.38, \mathrm{p}<0.001){ }^{[23]}$. A meta-analysis study by Lee and Song (2017) found that NLR was significantly higher in the systemic lupus erythematosus group than the control group (standard mean difference $[\mathrm{SMD}]=2.747,95 \%$ confidence interval $[\mathrm{CI}]=1.241 \sim 4.254, \mathrm{p} \quad<0.001)$. PLR was also significantly higher in the systemic lupus erythematosus group $(\mathrm{SMD}=1.564, \quad 95 \% \quad \mathrm{CI}=0.122 \sim 3.006$, $\mathrm{p}=0.034)^{[24]}$. It was concluded that NLR and PLR were found to be higher in patients with systemic lupus erythematosus and that there was a significant positive correlation between NLR/PLR activity and SLE.

\section{CONCLUSION}

The majority of the research subjects were women with an average age of 30 years, the youngest age 18 years, and the elderly 76 years. The prevalence of systemic lupus erythematosus was $0.01 \%$ with moderate, severe, and mild grades, approximately $39.3 \%, 33.3 \%$, and $27.5 \%$, respectively.

The ratio of neutrophil lymphocytes and platelet lymphocytes is related to the degree of activity of systemic lupus erythematosus. Neutrophil-lymphocyte ratio has a strong positive correlation with the platelet-to-lymphocyte ratio.

\section{Acknowledgement: None}




\section{Conflict of Interest: None}

\section{Source of Funding: None}

\section{Ethical Approval: Approved}

\section{REFERENCES}

1. Abd-Elhafeez, H.A., El-Meghawry, E.S., Omran, T.M., Alsayyad, M.M., Berengy, M.S. "Study of Neutrophil Lymphocyte Ratio and Platelet Lymphocyte Ratio as Inflammatory Markers in Systemic Lupus Erythematosus Egyptian Patients", Electronic Journal of Biology,.2017; 13(3):248-252.

2. Ali, A., Sayyed, Z., Ameer, M. A., Arif, A. W., Kiran, F., Iftikhar, A., et al. "Systemic Lupus Erythematosus: An Overview of the Disease Pathology and Its Management", Cureus. 2018; 10(9):3288.

3. Pons-Estel, G.J., Ugarte-Gil, M, F., Alarcón, G.S. 2017, "Epidemiology of systemic lupus erythematosus", Expert Rev Clin Immunol. 2017; 13(8): 799-814.

4. Oehadian, A., Suryadinata, H., Dewi, S., Pramudyo, R., Alisjahbana, B. "The Role of Neutrophyl Lymphocyte Count Ratio as an Inflammatory Marker in Systemic Lupus Erythematosus", Acta Medica Indonesiana The Indonesian Journal of Internal Medicine. 2013;45(3): 170-174.

5. Washio M, Kiyohara C, Ohta A. Epidemiology of Systemic Lupus Erythematosus, Lupus - New Advances and Challenges, Sophia Lionaki, IntechOpen, DOI: $\quad 10.5772 /$ intechopen.84146. 2019. Available from: https://www.intechopen.com/books/lupusnew-advances-andchallenges/epidemiology-of-systemic-lupuserythematosus

6. Yu, H., Jiang, L., Yao, L., Gan, C., Han, X., Liu, R., \& Su, N. "Predictive value of the neutrophil-to-lymphocyte ratio and hemoglobin in systemic lupus erythematosus", Experimental and therapeutic medicine. 2018; 16(2): 15471553.

7. Lehman T, Nuruzzaman F, Taber S. Chapter 8 - Systemic Lupus Erythematosus: Etiology, Pathogenesis, Clinical Manifestations, and Management. . Handbook of Systemic Autoimmune Diseases. Elsevier.2016; 11 173-189.
8. Tsokos, G.C., Lo, M.S., Costa Reis, P., Sullivan, K.E. "New insights into the immunopathogenesis of systemic lupus erythematosus", Nat. Rev. Rheumatol.2016;12( 12): 716-730.

9. Wang, L., Wang, C., Jia, X., Yang, M., Yu, J, "Relationship between Neutrophil-toLymphocyte Ratio and Systemic Lupus Erythematosus: A Metaanalysis", CLINICS.2020; 75:1450

10. Celikbilek, M., Dogan, S., Ozbakır, O., Zararsız, G., Kücük, H., Gürsoy, S., et al. 2013, "Neutrophil-lymphocyte ratio as a predictor of disease severity in ulcerative colitis", J Clin Lab Anal, .2013; 27(1): 72-6.

11. Turkmen, K., Erdur, F.M., Ozcicek, F., Ozcicek, A., Akbas, E.M., Ozbicer, A., et al. "Platelet-to-lymphocyte ratio better predicts inflammation than neutrophil-to-lymphocyte ratio in end-stage renal disease patients", Hemodialysis International.2013;17:391396.

12. Hao, X., Li, D., Wu, D. et al. The Relationship between Hematological Indices and Autoimmune Rheumatic Diseases (ARDs), a Meta-Analysis. Sci Rep 7.2017:10833.

13. Rees F, Doherty M, Grainge M, Davenport G, Lanyon P, Zhang W. The incidence and prevalence of systemic lupus erythematosus in the UK, 1999-2012. Ann Rheum Dis.2016; 75(1): 136-41.

14. Izmirly, P.M., Parton, H., Wang, L., McCune, W.J., Lim, S.S., Drenkard, C., et al. "Prevalence of Systemic Lupus Erythematosus in the United States: Estimates from a Meta-Analysis of the Centers for Disease Control and Prevention National Lupus Registries", Arthritis and Rheumatology.2021; 73: 991-996.

15. Zian, Z., Maamar, M., Aouni, M., Barakat, A., Nourouti, N.G., Aouad, R., Arji, N, Mechita, M.B, "Immunological and Clinical Characteristics of Systemic Lupus Erythematosus: A Series from Morocco", BioMed Research International.2018

16. Wichainun R, Kasitanon N, Wangkaew S, Hongsongkiat S, Sukitawut W, Louthrenoo W. Sensitivity and specificity of ANA and anti-dsDNA in the diagnosis of systemic lupus erythematosus: a comparison using control sera obtained from healthy individuals and patients with multiple medical problems. Asian Pac J Allergy Immunol, 2013; 4: 292-8. 
17. Qu, C. Zhang, J., Zhang, X., Du, J., Su, B, Li, H. 2018. Value of combined detection of anti-nuclear antibody, anti-double-stranded DNA antibody and C3, C4 complements in the clinical diagnosis of systemic lupus erythematosus. Experimental and Therapeutic Medicine. 2018;17(2).

18. Ismunandar, Syahriani, F., Faridin, H.P., Bakri, S., Kasim, H., Harjianti, T., et al. "Neutrophyl Lymphocyte Ratio and Platelet Lymphocyte Ratio Associated With Disease Activity in Patients with Systemic Lupus Erythematosus", European Journal of Molecular and Clinical Medicine. 2020; 7 (8): 978-984.

19. Smirnova, E.V., Krasnova, T.N., Proskurnina, E.V., Mukhin, N.A. 'Rol' disfunktsii neǐtrofilov $\mathrm{V}$ patogeneze sistemnoĭ krasnoî volchanki [Role of neutrophil dysfunction in the pathogenesis of systemic lupus erythematosus]", Ter Arkh, 2017; 89(12): 110-113.

20. Soliman, W.M., Sherif, N.M., Ghanima, I.M., \& EL-Badawy, M.A., "Neutrophil to Lymphocyte and Platelet to Lymphocyte Ratios in Systemic Lupus Erythematosus: Relation With Disease Activity and Lupus Nephritis", Reumatología Clínica,2020; 16(4):255-261.

21. Han, B.K., Wysham, K.D., Cain, K.C., Tyden, H., Bengstsson, A.A., Lood, C.
"Neutrophil and lymphocyte counts are associated with different immunopathological mechanisms in systemic lupus Erythematosus", Lupus Science \& Medicine.2020; 7: e000382.

22. Qin, B. et al. Neutrophil to lymphocyte ratio (NLR) and platelet to lymphocyte ratio (PLR) were useful markers in assessment of inflammatory response and disease activity in SLE patients. Mod Rheumatol. 2016; 26(3): 372-6.

23. Li, L., Xia, Y., Chen, C., Cheng, P., Peng, C. "Neutrophil-lymphocyte ratio in systemic lupus erythematosus disease: a retrospective study", Int J Clin Exp Med. 2015; 8(7): 11026-31.

24. Lee, Y. H., Song, G. G. 2017, “Association of Neutrophil to Lymphocyte Ratio, Platelet to Lymphocyte Ratio, and Mean Platelet Volume with Systemic Lupus Erythematosus Disease Activity: A Metaanalysis", Journal of Rheumatic Diseases. 2017;24(5): 279.

How to cite this article: Fikri I, Zubir Z, Ginting AW. The relationship between neutrophil lymphocyte ratio and platelet lymphocyte ratio to degree of activity in systemic lupus erythematosus. International Journal of Research and Review. 2021; 8(10): 374-382. DOI: https://doi.org/10.52403/ijrr.20211050 